LETTER TO THE EDITOR

\title{
Foundations of undergraduate training
}

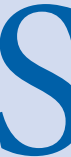
ir, I read with interest the recent themed edition of BDJ In Practice, which gave a fascinating perspective on the status of implant dentistry in the United Kingdom with a range of thought-provoking articles.

From my perspective I placed my first dental implant in 2001 (closely mentored at The Eastman) and since then have developed a very broad experience of both surgical and prosthodontic elements of implant dentistry. As I now work in a dental school, I noted the highlighted comment 'undergraduate dentistry degrees providing very limited to no training. The GDC states that dental students should 'be familiar with dental implants as an option in replacing missing teeth' which considering the future cohort of patients current students and those newly-qualified will be treating, has always seemed entirely inadequate.'

When I reflect on my own undergraduate training (qualified University of Edinburgh 1993), even though dental implants as such were not part of the curriculum at that stage, a huge part of my planning and thought process in implant dentistry in 2021 relates to what I was taught as an undergraduate! By this I would highlight the excellent training I had in understanding anatomy, bone biology, hard and soft tissue healing, individual differences in inflammatory responses, manual dexterity, consent, planning for complications and failure, control of active dental disease and understanding a range of treatment modalities should always be considered.

In addition to many other areas relevant to implant dentistry, these continue to be key elements of undergraduate training across the UK, and in my opinion should continue to remain the foundation stone of skills escalation in implant dentistry.

Ewen McColl Peninsula Dental School, Plymouth

\section{Scottish Government COVID-19 inquiry: Dental profession must be better supported in a future pandemic}

Dental professionals must receive better support for their physical, emotional and financial wellbeing in a future pandemic situation, to ensure patients continue to get the best possible care and treatment and avoid long-term impacts on the profession.

In its submission to the Scottish

Government's inquiry into the handling of the COVID-19 pandemic, Dental Protection said the dental profession desperately needed priority PPE, support measures to mitigate potential financial viability issues due to safety protocols limiting access to dental services, and consistent guidelines.

The organisation said the Scottish Dental Clinical Effectiveness Programme (SDCEP) provided well received clinical guidance at a time when dental professionals around the world sought clarity about operating procedures, and this was a positive learning for the future.

However, Dental Protection said there was an overall sense that dentistry has been 'overlooked' throughout the COVID-19 pandemic, and a number of lessons must be learnt should we face a further pandemic.

Helen Kaney, Dental Protection Head of Dental Services, Scotland, said: 'Dental professionals have faced significant challenges as a result of the pandemic. The clinical challenges have centred on availability of the required PPE, virus transmission risk and restrictions of the type of clinical interventions that could be undertaken for patients. There have also been and remain, significant financial challenges which may impact on the long-term provision of NHS dentistry in Scotland.

'These challenges were enhanced by a sense that dentistry has been overlooked throughout much of the pandemic. Dental professionals are well used to dealing with and managing risk, but the scale of risk and uncertainty for dentistry throughout this time has been unprecedented.

'Dental Protection has throughout the pandemic been providing dentolegal advice to those dental professionals grappling with these new challenges, to enable them to practise in different ways, comply with guidelines and to help them protect their wellbeing.

'But the profession as a whole needs greater support from the outset should we face a pandemic situation in the future - this includes swift publication of clear, consistent guidelines, appropriate PPE as a priority, and effective financial support measures to protect the financial stability of practices and ensure that patients can continue to receive optimal care within the safety protocols.

'Addressing the shortfalls from this crisis will be vital in ensuring patients continue to get the best possible care in any future pandemic, and in safeguarding our profession. We hope the challenges we have raised will be considered as part of the inquiry'

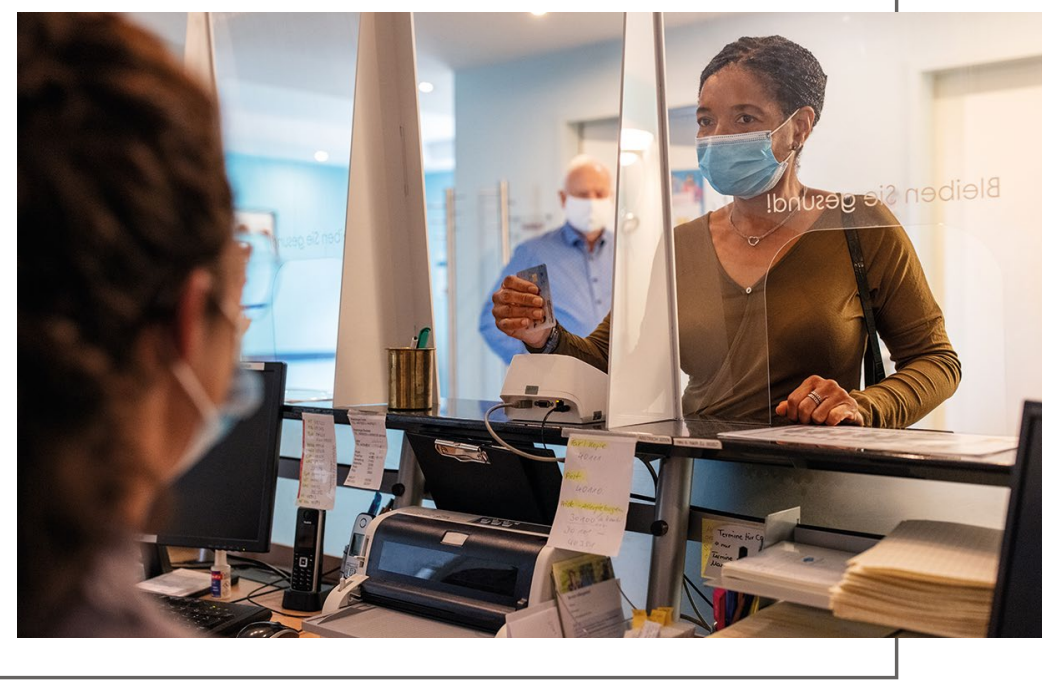

